\title{
Recalibrated Scales: \\ The Use of Low-dose Isotretinoin in a Case of Epidermolytic Ichthyosis-NPS1 in a Filipino Child
}

\author{
Erickah Mary Therese R. Dy, MD, Shahara Abalos-Babaran, MD, \\ Monette R. Faner, MD and Carmela Augusta F. Dayrit-Castro, MD \\ Department of Dermatology, Philippine General Hospital, University of the Philippines Manila
}

\begin{abstract}
Epidermolytic Ichthyosis (EI) is a rare non-syndromic keratinopathic ichthyosis without definitive treatment. This is a case of El in a 5-year-old Filipino female who presented with hyperkeratotic scales sparing the palms and soles. Histopathology revealed epidermolytic hyperkeratosis. A trial of treatment with isotretinoin $0.3 \mathrm{mg} / \mathrm{kg} / \mathrm{day}$, together with keratolytic agents, urea lotion and lactic acid lotion, resulted in a marked decrease in the thickness of the scales and odor. Interestingly, rebound effects were noted at $0.6 \mathrm{mg} / \mathrm{kg} / \mathrm{day}$. Taking into account that El presents with more skin fragility compared to non-EHK ichthyosis, the authors surmise that there may be a smaller treatment window for patients with El, which is notably lower than recommended for ichthyosis in general.
\end{abstract}

Key Words: epidermolytic hyperkeratosis, isotretinoin

\section{INTRODUCTION}

Epidermolytic Ichthyosis (EI), previously called bullous congenital ichthyosiform erythroderma (BCIE), is a rare autosomal dominant keratinopathic ichthyosis that presents with blistering and erythema at birth, subsequently replaced by hyperkeratosis starting in the first months of life. ${ }^{1}$ It has a prevalence of 1 in about 300,000 persons, and presents as a sporadic case from spontaneous mutations in about half of the patients. ${ }^{1}$ The diagnosis of EI remains mainly clinical, supplemented by histopathology showing epidermolytic hyperkeratosis (EHK), a histopathologic term for the typical light microscopic findings in EI including hyperkeratosis, hypergranulosis, and suprabasal vacuolar degeneration. ${ }^{2}$ Genetic testing has also been done for EI, and may give a definitive diagnosis, but this is not widely available. ${ }^{2}$ The clinical presentation of EI can vary and these have been classified into six subtypes, three of which present with severe palmoplantar hyperkeratosis, and the other three without palmoplantar involvement. ${ }^{3}$ The prognosis is generally good, with blistering and superimposed infection becoming less frequent with age. ${ }^{1}$ However, clinical features and symptoms that remain until adulthood such as pruritus and fissuring, together with the cosmetic concerns, may lead to reduced quality of life. ${ }^{2}$ Currently, there is no definitive treatment for EI, but emollients, keratolytic agents, and retinoids have been used to control the hyperkeratosis. ${ }^{4}$ In this report, we present a case of a 5-year-old Filipino female with good response to trial of treatment with low-dose

isotretinoin at $0.3 \mathrm{mg} / \mathrm{kg} / \mathrm{day}(\mathrm{mkd})$.

Corresponding author: Erickah Mary Therese R. Dy, MD

Department of Dermatology

Philippine General Hospital

University of the Philippines Manila

Taft Avenue, Ermita, Manila 1000, Philippines

Email: erdy@up.edu.ph 


\section{CASE DESCRIPTION}

This is the case of a 5-year-old Filipino girl with the chief complaint of generalized scaling and thickening of the skin. A three-generation genogram of the patient's family revealed a non-consanguineous marriage and the presence of the condition only in the patient. Patient was born full term via spontaneous vaginal delivery by a then 31-year-old mother (G2P1 1001) at a lying-in clinic, assisted by an obstetrician. Pregnancy and delivery course were unremarkable. There was no exposure to teratogens in utero.

At birth, generalized xerosis and lower extremity erosions were noted, without any intact blisters appreciated. At 3-4 months of age, patient developed thick brown scales and thickening of skin. Pruritus, malodor and skin fragility subsequently developed. Initial consult was done with a local health care provider when the patient was three years of age. Emollients were recommended with partial relief of pruritus.

On physical examination, patient's height and body mass index (BMI) were noted to be normal for age. Dermatologic examination revealed generalized scaling (Figure 1A) with multiple, well-defined light to dark brown papules and plaques with thick adherent scales. Plaques on the trunk, extremities, and external genitalia had a cobblestone-like pattern (Figure 1B), with corrugated pattern over the joints (Figure $1 \mathrm{C}$ ), and spared the face, palms, and soles (Figure 1D). Patient was also noted to have a distinct odor.

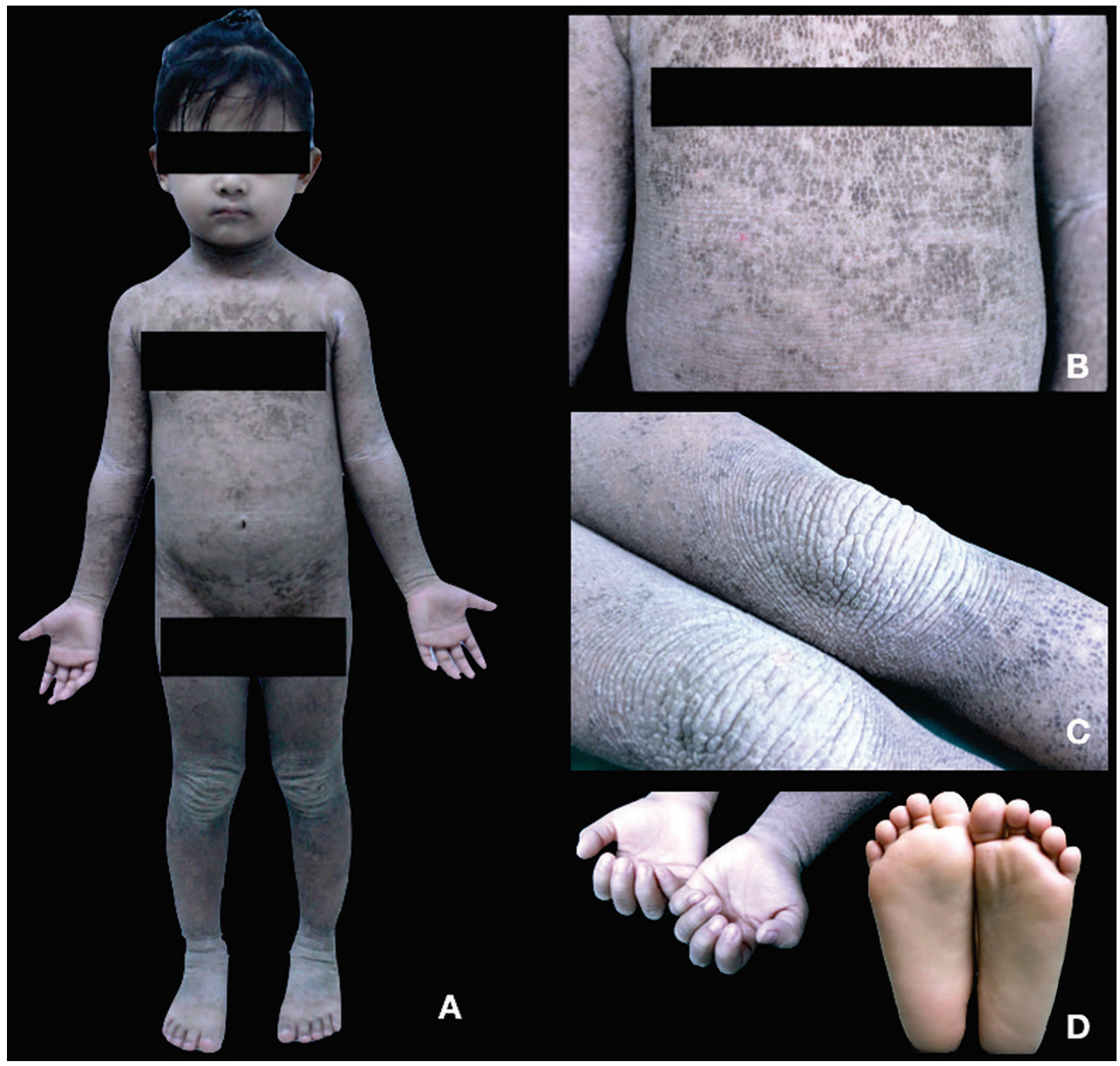

Figure 1. Clinical findings of epidermolytic ichthyosis NPS1. Generalized scaling (A) with cobblestone-like pattern on the trunk (B), with corrugated pattern over the knees (C), and sparing the palms and soles (D). 


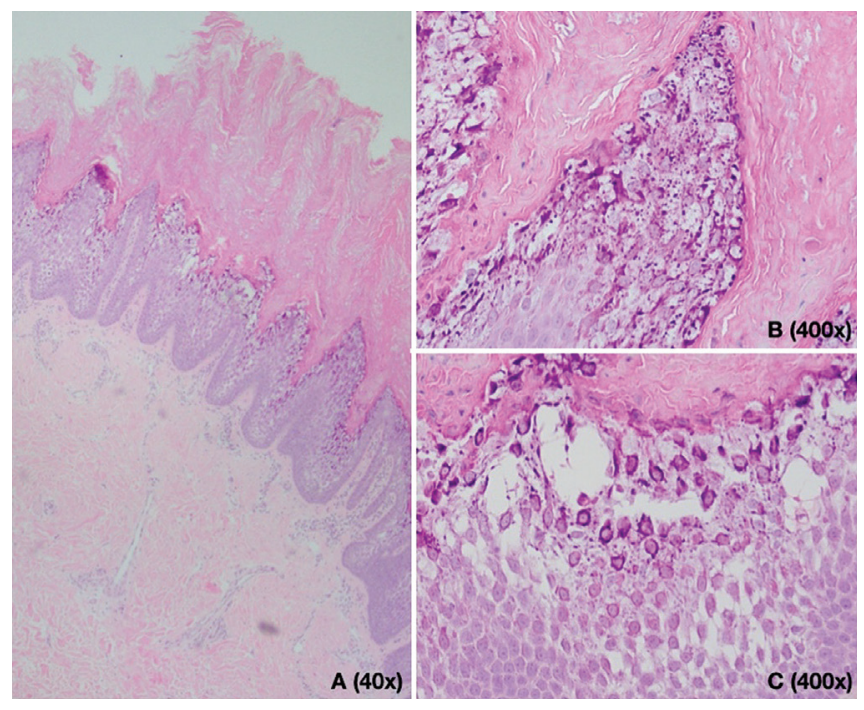

Figure 2. Histopathologic review of 4-mm punch biopsy taken from the lower back (Hematoxylin and eosin). These sections show hyperkeratosis, vacuolar changes involving the suprabasilar epidermis (A) most marked at the granular layer (B), and intraepidermal blisters (C).

A 4-mm punch biopsy taken from the lower back of the patient showed hyperkeratosis and vacuolar changes involving the suprabasilar epidermis (Figure 2A) most marked at the granular layer (Figure 2B). Small intraepidermal blisters secondary to the vacuolar changes were also observed (Figure 2C).

Based on the clinical presentation and histopathologic findings, the patient was diagnosed with epidermolytic ichthyosis with no hyperkeratosis of the palms and soles (EI-NPS). The patient was prescribed urea $10 \%$ with lactic acid 10\% lotion twice a day for 4 weeks while awaiting results of laboratory tests (fasting blood glucose, lipid profile, liver enzymes, and complete blood count), before starting a trial of treatment with a systemic retinoid.

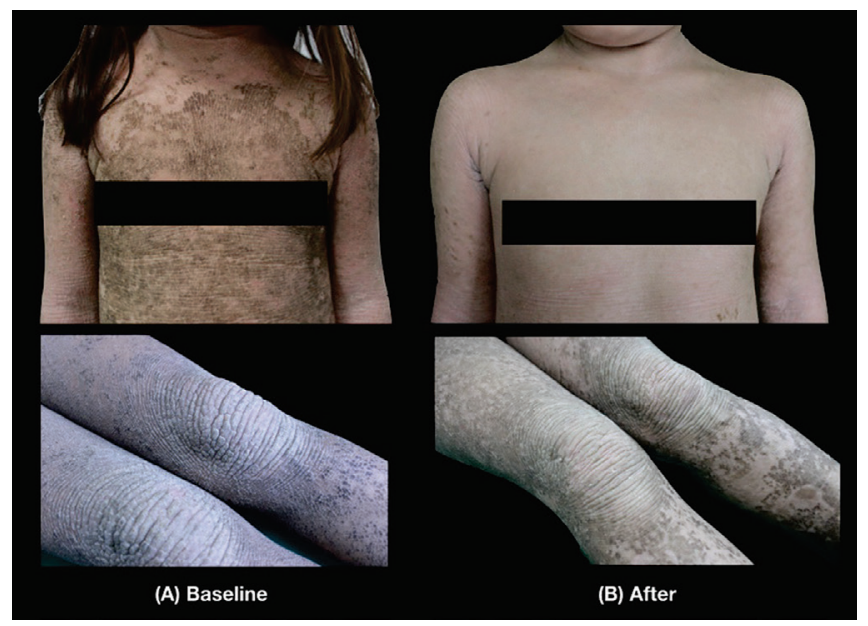

Figure 3. Epidermolytic Ichthyosis before (A) and after (B) 1 month of treatment with $0.3 \mathrm{mkd}$ isotretinoin and lactic acid $10 \%$ lotion twice a day.

With normal baseline laboratory results, the patient was started on isotretinoin at $0.3 \mathrm{mkd}$. Isotretinoin was used due to the unavailability of acitretin in the country. Lactic acid $10 \%$ lotion was continued with once weekly bleach baths. On follow up after one month, there was marked decrease in the hyperkeratosis and odor (Figures 3A and 3B, Figures $4 \mathrm{~A}$ and $4 \mathrm{~B}$ ). Repeat laboratory results after one month of isotretinoin were normal.

Given the improvement seen, the dose of isotretinoin was increased to $0.6 \mathrm{mkd}$. Topicals were shifted to twice daily urea $10 \%$ lotion and weekly bleach baths were continued. On follow-up after one month, the scales increased in number (Figure 4C). Laboratory results were normal, except for an increase in aspartate aminotransferase (AST) to 1.5 times above normal. The patient's guardian did not note cheilitis nor xerosis with the increase in dose of isotretinoin.

Besides the change in dose, other variables that could have affected the outcome at this stage were the change in brand of isotretinoin and the shift from lactic acid to urea

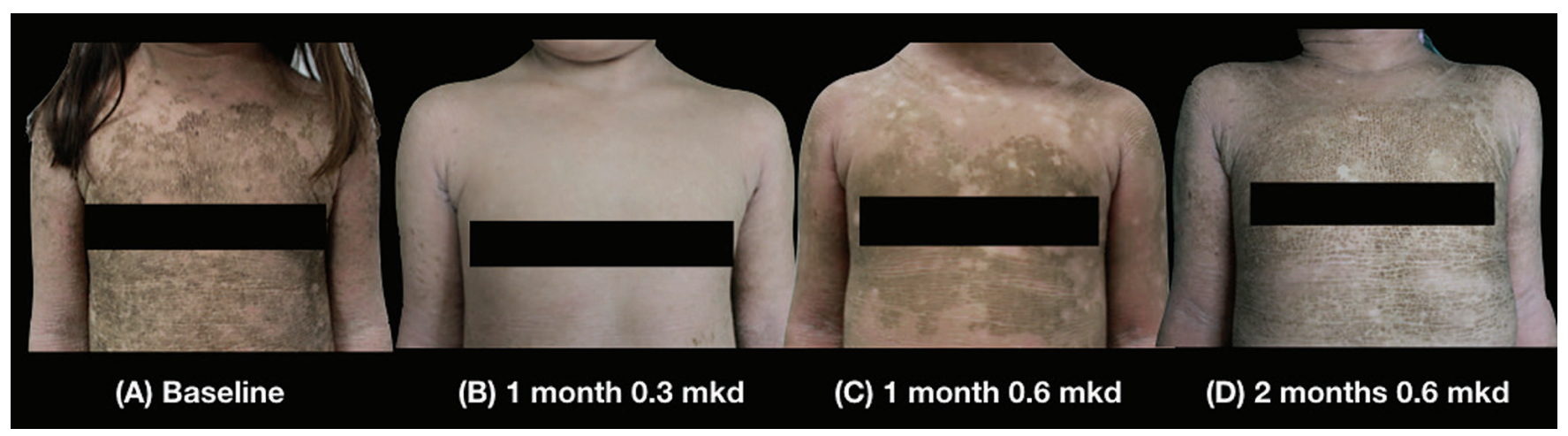

Figure 4. Epidermolytic ichthyosis at baseline (A), after 1 month 0.3 mkd (B), after 1 month of 0.6 mkd (C), and after 2 months $0.6 \mathrm{mkd}(\mathrm{D})$ of isotretinoin. 
lotion. Given these, the dose of isotretinoin was maintained at $0.6 \mathrm{mkd}$, reverting back to the initial isotretinoin brand used. Topicals were shifted back to lactic acid 10\% and weekly bleach baths were maintained. On follow-up again after 1 month, the patient still had more scales as compared to the results of the first month of treatment (Figure 4D). Laboratory results were normal, except for a further increase in AST.

Given the response, the isotretinoin dose was reverted back to $0.3 \mathrm{mkd}$. Topicals and weekly bleach baths were maintained. Subsequent follow ups were interrupted due to the coronavirus-19 disease (COVID-19) pandemic.

\section{DISCUSSION}

Epidermolytic Ichthyosis (EI), previously called bullous congenital ichthyosiform erythroderma (BCIE), is a rare autosomal dominant keratinopathic ichthyosis. It is caused by mutations in genes coding for keratin 1 (KRT1) or keratin 10 (KRT10). ${ }^{5}$ Keratins 1 and 10 are expressed in the differentiated suprabasal cells of the epidermis. ${ }^{6}$ The general structure of keratins consists of a central rod domain bounded by highly conserved regions that play an important role in keratin polymerization. ${ }^{7}$ It is crucial because the keratin molecules need to polymerize to form the intermediate filament, which forms the cytoskeleton of the epidermal cells, imparting structural support and stability to the epidermis. ${ }^{7,8}$ Thus, the clinical presentation of epidermolytic ichthyosis can vary, depending on what part of the keratin gene was mutated, such that mutations affecting the highly conserved domains lead to severe phenotypes. ${ }^{5}$ The mutant keratins aggregate and clump, leading to the collapse of the intermediate filament network and to endoplasmic reticulum stress, ultimately leading to apoptosis. ${ }^{9}$ Histologically, this translates to the vacuolar degeneration seen in the upper epidermis. Severe palmar and plantar involvement is associated with mutations in KRT1, while no severe palmoplantar involvement is associated with KRT10 mutations. ${ }^{1}$ This may be explained by the presence of keratin 9 (KRT9), which occurs only in the suprabasal epidermis of palmar and plantar skin, and reflects the redundancy of KRT9 and KRT10 in the said areas. ${ }^{1}$

The heterogeneity of the EI phenotypes has been simplified into six classifications, with three types showing palmoplantar hyperkeratosis and three types without. ${ }^{3}$ Among the phenotypes described, EI-NPS 1 is most compatible with our patient. EI-NPS 1 presents with generalized hyperkeratosis with yellow-to-dark brown thick scales, sparing the palms and soles. ${ }^{3}$

The diagnosis of EI remains mainly clinical, supported by the histopathologic finding of epidermolytic hyperkeratosis (EHK) which consists of hyperkeratosis, hypergranulosis and suprabasal vacuolar degeneration. ${ }^{2}$ All of these histological findings were observed in this case. Genetic testing showing mutations in keratin 1 (KRT1) or keratin 10 (KRT10) also support the diagnosis. ${ }^{5}$
EHK, however, is not specific for EI, and may also be seen in other keratinopathic ichthyoses. ${ }^{10}$ Among the other keratinopathic ichthyoses, the closest differential to our case would be Ichthyosis Hystrix Lambert (IHL), which also presents with generalized hyperkeratosis with thick brown scales, sparing the palms and soles. However, erosions at birth, pruritus, and formation of blisters after minor trauma, all observed in EI, are not seen in IHL. ${ }^{10,11}$

EI presents with blistering and erythema at birth, subsequently replaced by hyperkeratosis starting in the first months of life. Patients also develop a characteristic odor caused by bacterial superinfection. Cutaneous findings include generalized hyperkeratosis with thick brown scales, often with characteristic corrugated pattern accentuated in some areas, such as the knees of our patient. The prognosis is generally good, with blistering and superimposed infection becoming less frequent with age. ${ }^{1}$ However, clinical features and symptoms that remain until adulthood such as pruritus and fissuring, together with cosmetic concerns, may lead to reduced quality of life. ${ }^{2}$

In general, management of ichthyosis includes proper nutrition due to the higher nutritional requirements brought about by a hypermetabolic state caused by the large turnover of scales and energy loss from impaired barrier function. Heat intolerance precaution, such as having water spray bottles or ice packs, is advised as ichthyosis patients may have decreased sweating with heat intolerance. ${ }^{1}$

For epidermolytic ichthyosis, the mainstays of treatment are emollients and bathing, supplemented by keratolytic agents, antimicrobials, and retinoids. For bathing habits, soaking for long periods, up to an hour, supplemented by the gentle use of rough textured sponges or abrasives, leads to skin hydration and scale removal. Bleach baths minimize bacterial overgrowth. Keratolytic agents include lactic acid and urea. In our case, we chose to give lactic acid lotion and urea $10 \%$ lotion, alternating every four weeks, to prevent any side effects from chronic use of topical medications. Salicylic acid may also be used, but only for limited involvement as systemic absorption may cause salicylism. Though patients with EI are prone to bacterial and fungal infections, the use of prophylactic antimicrobials is not recommended, as it has been shown to promote the growth of resistant bacteria and Pseudomonas aeruginosa. Among the topical retinoids, tazarotene is more widely used for EI. Adverse effects include erythema, pruritus, and burning sensation. Lastly, oral retinoids can be used, starting at low doses, and escalating as tolerated as this can lead to increased skin fragility and erythema in EI. A study done in 2008 investigated the effects of acitretin in children with disorders of keratinization. Four EI patients were included in the study population. The study showed that increasing skin fragility, as a side effect, was most commonly seen in the EI population, in comparison to the other types of ichthyosis. ${ }^{12}$ The increased skin fragility is postulated to be due to a decrease in desmosomes seen in patients receiving systemic retinoids. ${ }^{13}$ In literature, the 
common dose for acitretin is $0.5 \mathrm{mkd}$, while for isotretinoin, approximately $1 \mathrm{mkd}$. In our patient, a better response was noted at a much lower dose of $0.3 \mathrm{mkd}$ together with the use of lactic acid $10 \%$ compared to $0.6 \mathrm{mkd}$ with either urea $10 \%$ or lactic acid 10\%. The lower dose is surmised to be within the window of response where the effects of systemic retinoids is maximized, with minimum induction of skin fragility. Toxicities are similar for acitretin and isotretinoin, with adverse effects including cheilitis, lipid and liver function test derangements, and hyperostosis with prolonged use. ${ }^{4}$

Literature regarding the skeletal side effects is conflicting. Earlier studies showed severe skeletal side effects of isotretinoin. A 1982 case report showed premature epiphyseal closure in a child with epidermolytic ichthyosis; however, doses were very high and reached $4.5 \mathrm{mkd}$, and were even supplemented with $5000 \mathrm{IU}$ of vitamin A. ${ }^{14}$ In a case series published in 1984, 6 of 8 patients aged 5 to 26 years developed skeletal hyperostoses with isotretinoin treatment at 1 to $2.9 \mathrm{mkd}$, with an average dose of $2.0 \mathrm{mkd}$, for 1 year of therapy. ${ }^{15}$ They noted that the changes may regress after discontinuation of therapy. ${ }^{15}$ More recent publications, however, report long term safe use of retinoid therapy, with a suggested dose of isotretinoin at $1 \mathrm{mkd} .{ }^{16}$ Baseline radiographs are suggested, once the patient decides that the therapy is well-tolerated and desires chronic therapy. ${ }^{16}$ A baseline skeletal survey was planned for the patient if they decided to push through with long-term therapy based on the trial of treatment.

Future plans for the patient include genetic testing for KRT 10 mutation, which is the likely gene affected in EI cases with palmoplantar sparing.

\section{CONCLUSION}

In patients with suspected ichthyosis, a complete history and physical examination is vital. The diagnosis remains mainly clinical with disease classifications still evolving. Since there is currently no definitive cure, the therapeutic plan for patients with ichthyosis should take into account not just the symptoms but the general implications of their skin barrier defects, such as heat intolerance, hypermetabolic state, and possible reduced quality of life, as well as possible side effects of medications with prolonged use. In EI, high doses of oral retinoids can lead to increased skin fragility. This case suggests a very small window of good response to oral retinoids, seen at a dose that is significantly lower than recommended.

\section{Statement of Authorship}

All authors participated in the data collection and analysis and approved the final version submitted.

\section{Author Disclosure}

All authors declared no conflicts of interest.

\section{Funding Source}

This study has no funding support.

\section{REFERENCES}

1. Choate KA, Milstone LM. The ichthyoses. In: Kang S, Amagai M, Bruckner AL, Enk AH, Margolis DJ, McMichael AJ, Orringer JS, eds. Fitzpatrick's Dermatology, 9th ed. New York: McGraw-Hill Education; 2019. pp. 775-815.

2. Rout DP, Nair A, Gupta A, Kumar P. Epidermolytic hyperkeratosis: clinical update. Clin Cosmet Investig Dermatol. 2019 Dec; 12: 333-44.

3. Digiovanna JJ, Bale SJ. Clinical heterogeneity in epidermolytic hyperkeratosis. Arch Dermatol. 1994 Aug; 130(8):1026-35.

4. Mathes EF, Spring S, Friedland R, Paller AS. Hereditary disorders of cornification. In:TengJMC, Marqueling AL, Benjamin LT, eds. Therapy in pediatric dermatology: Management of pediatric skin disease. Switzerland: Springer International Publishing; 2017. pp. 51-68.

5. Kono M, Fukai K, Omura R, Sugawara K, Tsuruta D, Sugiura K, Akiyama M. A case of epidermolytic ichthyosis showing a very mild phenotype due to a novel tail extension mutation in KRT10. J Eur Acad Dermatol Venereol. 2016; 31(2):1-3.

6. Moll R, Divo M, Langbein L. The human keratins: biology and pathology. Histochem Cell Biol. 2008; 129(6): 705-33.

7. Jacob JT, Coulombe PA, Kwan R, Omary MB. Types I and II Keratin Intermediate Filaments. Cold Spring Harb Perspect Biol. 2018 May; 10(4):1-17.

8. Wang B, Yang W, Mckittrick J, Meyers MA. Keratin: Structure, mechanical properties, occurrence in biological organisms, and efforts at bioinspiration. Prog Mater Sci. 2016 Jul; 76:229-318.

9. Takemoto K, Makino T, Mizawa M, Kubo Y, Shimizu T. Missense mutation $\mathrm{Y} 449 \mathrm{H}$ of the $\mathrm{K} 10$ gene in a patient with severe epidermolytic ichthyosis. Eur J Dermatol. 2019 Mar; 29(2):227-8.

10. Oji V, Tadini G, Akiyama M, Bardon CB, Bodemer C, Bourrat E, et al. Revised nomenclature and classification of inherited ichthyoses: Results of the First Ichthyosis Consensus Conference in Sorèze 2009. J Am Acad Dermatol. 2010 Oct; 63(4):607-41.

11. Wang WH, Li LF, Zhang Q, Yang SM, Jiang W, Wang YY, et al. Ultrastructural features of ichthyosis hystrix strongly resembling Lambert type. Br J Dermatol. 2007; 156(5): 1027-31.

12. Zhang XB, Luo Q, Li CX, He YQ, Xu X. Clinical investigation of acitretin in children with severe inherited keratinization disorders in China. J Dermatolog Treat. 2008; 19(4):221-8. doi: 10.1080/09546630801955150.

13. Williams ML, Elias PM. Nature of skin fragility in patients receiving retinoids for systemic effect. Arch Dermatol. 1981 Oct; 117(10):611-9.

14. Milstone LM, Mcguire J, Ablow RC. Premature epiphyseal closure in a child receiving oral 13-cis-retinoic acid. J Am Acad Dermatol. 1982 Nov; 7(5):663-6.

15. Pennes DR, Ellis CN, Madison KC, Voorhees JJ, Martel W. Early skeletal hyperostoses secondary to 13-cis-retinoic acid. Am J Roentgenol. 1984 May; 141(5): 979-83.

16. Digiovanna JJ, Mauro T, Milstone LM, Schmuth M, Toro JR. Systemic retinoids in the management of ichthyoses and related skin types. Dermatol Ther. 2013 Jan; 26(1):26-38. 